\title{
II. Ueber einen Fall von Lues universalis, insbesondere des Centralnervensystems.
}

Von Dr. M. Jastrowitz. ${ }^{2}$ )

M. H.! Der Fall von Lues universalis, den ich Thnen qu demonstrixen mir gestatte, betrifft namentlich das Centralnervensystem an verschiedenen Orten, und ich bedaure nur, dass ich nicht sămmtliche Präparate zưr Stelle haben kann; dieselben würden insgesammt Ihr Interesse erregen. Sie entstammen einer 42 jährigen Frau, ehemaligen Gattin eines Majors, der im Feldzuge fiel, mit dem sie zwei gesunde Kinder erzeugt hatte. Sie ging eine zweite Whe ein, in der sie syphilitisch inficirt worden ist. Es steht sicher fest, dass in den 70er Jahren, etwa 1877, ihr zweiter Gatte in diversen Krankenhäusern an schwerer Syphilis behandelt wurde. Die Frau lebte auf dem Lande und scheint sich zuerst über die Natur ibres Leidens nicht klar geworden za sein. Es ist auch nicht ganz aufgeklärt, wann sie zuerst luetisch erkrankte. Im Februar 1881, so gab ein Freund ihrer Familie an, litt sie am Gaumen, und die Nase sank ein. College Böcker macht mir nachträglich die Mitthéilung, dass er sie 1882 an einer, sèiner damalígen Diagnose nach, luetischen Affection der Nase behandelt habe. Seit Ende 1885 traten zeitweilige Anschwellung der Nase mit foetidem Ausfluss aus derselben und starke Kopfcongestionen auf. Eine ing Frühjahr 1886 dagegen gebrauchte 8chmierkur, die sile anscheinend unvollkommen and schlecht selber an sich ausführte, war dagegen ohne Eirfotg. Man fand sie

1) Zeitschr. f. physiolog. Chemie von Hoppe-Seyler Bd. p. 145

2) Vortrag gehalten im Verein für innere Medicin. 
mehrmals in ihrer Wohnung fast besinnungslos, zuweilen Unsinn schwatzend; im Sommer, seit Mitte Juli 1886, stellten sich Schwindel, heftige Kopfschmerzen, besonders zur Nachtzeit, und Sehstörungen ein.

Am 4. September v. J. wurde sie in die Maison de santé aufgenommen wegen einer Psychose. Diese Psychose bestand in einem ängstlich erregten Zustand, in allgemeiner Verworrenheit, nebst Sinnestäuschungen des Gesichts und des Gehörs. Sie war weder über die Zeit, noch über ihren Aufenthaltsort orientirt, suchte und tappte überall umher, hörte die Stimmen ihrer Söhne, denen sie Aufträge ertheilte, besprach in lauten Selbstunterhaltungen vorzugsweise Vermögensverhältnisse, glaubte sich übervortheilt und bestohlen.

Schon draussen waren von dem behandelnden Arzt Dr. Bernheim Sehstörungen wahrgenommen worden; es war die Diagnose einer luetischen Cerebralaffection und Neuroretinitis gestellt. In der That fand sich bei der ophthalmoskopischen Untersuchung eine Neuroretinitis; die Papille war getrübt, auch die Retina in leichtem Grade an verschiedenen Stellen. Es bestanden drei Blutaustretungen in der Nähe der Papille und an den Gabelungen zweier Gefässe. Die Pupillen waren weit und reagirten etwas träg. Was Motilität und Sensibilität anlangte, so bot die Patientin garnichts. Die sonstige objective Untersuchung ergab: Schwellung derHals-, Nacken-, Inguinaldrüsen, Haut rein, runder Defect im harten Gaumen nahe den Schneidezähnen, foetider, eitriger Ausfluss aus der Nase, deren Rücken eingesunken, Unebenheiten an dem kleinen runden Schädel, eine besonders auffällige oberhalb der Glabella, aber äusserlich keine Narben, an der rechten Tibia eine kleine Auftreibung.

Die Kranke wurde zuerst mit Jodkali behandelt, und besserte sich nach jeder Richtung bald, so dass sie am 11. September bereits eine Zeitung zu lesen vermochte. Hernach instituirte ich eine Schmierkur und habe dieselbe mit Unterbrechungen fast 8 Wochen lang durchgeführt, bis die Kranke mir erklärte, sie wolle sich nicht mehr schmieren lassen. Sie hat im Ganzen 147 Gramm Unguent cinereum verbraucht. In der That war sie soweit besser geworden, dass ich nach nochmaliger Untersuchung, die ich vor ihrer Entlassung vornahm, bestimmt sagen kann, es sei keine Motilitäts- und Sensibilitätsstörung vorhanden gewesen. Das Sehen war etwas beeinträchtigt, indem die Sehschärfe abgenommen hatte, doch fertigte sie mit einer Brille feine Handarbeiten an. Geistig zeigte sie sich vollkommen normal, sie motivirte die Nothwendigkeit der Unterbrechung ihrer Kur, da ich ihr die möglichen Folgen vorstellte, mit ihren eigenartigen Verhältnissen in einer Weise, dass ich mich von ihrer von jeher bestandenen Umsicht und geistigen Begabung und von ihrem charaktervollen Wesen überzeugen konnte.

Nachdem die Pat. das Krankenhaus am 14. Novbr. verlassen hatte, ging es ihr, soweit ich benachrichtigt wurde, ganz gut bis etwa Weihnachten v. J. Dann stellte sich wieder eine eigenthümliche Zerstreutheit nebst Sehstörungen ein. Diese Zerstreutheit nahm zu, sie wurde verworren und, ich weiss nicht aus welchem Grunde, bettlägerig. Sie sollüber Kopfschmerzgeklagt und, man konnte nicht entscheiden ob aus Zerstreutheit oder wegen Sehstörung, Gegenstände verwechselt z. B. in dem Sahnentopf auf dem Frühstückstische die Zuckerdose erblickt haben. - Sie sprach dann unverständlich, konnte nicht recht schlucken, so dass sie in der letzten Zeit vor der Aufnahme in die Anstalt am 1. Febr. 1887 kaum mehr Nahrung zu sich genommen hat. Wir fanden sie in einem sehr kläglichen Zustande. Sie lag unbeholfen da, doch konnte man isolirte Lähmungen an Gesicht und Extremitäten nicht wahrnehmen. Die Sprache war sehr unvollkommen und unverständlich, sie nannte aber noch den Namen des Arztes, der sie behandelt hatte. Die Herzaction war sehr schlecht, der Puls kaum fühlbar. Sie konnte nicht schlucken, Temperatur 38,1. Der saure und getrübte Urin enthielt eine Spur Eiweiss, keine Cylinder, spärliche Schleimkörperchen, viel harnsaure Salze. In der Nacht vom 2-3. Febr. bekam Patientin Collaps und starb am Morgen früh.

Die Section hat ein nach vielen Beziehungen interessantes Resultat ergeben. Schon bei Lebzeiten war, wie ich berichtete, der kleine Schädel der Pat. aufgefallen. Derselbe zeigte eine enorme Hyperostosis generalis, am stärksten am Stirnbein wo der Schädel $1,75 \mathrm{~cm}$ dick war. Die Diploë ist allenthalben völlig geschwunden, die Nähte sind verstrichen. Wenn man von oben her, nachdem das Hirn herausgenommen ist, in den Schädel hineinsieht. hat es gleichsam den Anschein, als ob man in Vogelperspective auf ein Gebirge en miniature sieht, so sehr sind die physiologischen Erhöhungen, alle Processus und Juga cerebralia spitz hypertrophirt. Ausserdem finden sich an der Innenfläche allenthalben kleine weizenkorngrosse Exostosen. Von aussen, oberhalb der Glabella sehen Sie diese über 3 Centimeter grosse und charakteristische Narbe am Stirnbein (Demonstration), die ohne weiteres die Krankheit als syphilitische bewahrheitet, welche entstanden ist unterhalb einer glatten intacten äusseren Bedeckung.
Was das Gehirn angeht, so war an der Dura nichts bemerkenswerth und, abgesehen von einer geringen intermeningealen Blutung oberhalb der Partes orbit. der 2. linken und 1. rechten Stirnwindung, ebensowenig an der Pia, keine Verwachsung, die Gefässe zart, nur die Carotis interna auf dem Querschnitt etwas verdickt. Die Substanzen der Hemisphären waren ziemlich weich, mässig blutreich. Bei einem Längsschnitt durch die rechte Hemisphäre gelangte man auf einen Heerd, der den vorderen und grössten Theil des Nucleus caudatus vom Corpus striatum, den vordersten Theil des Nucleus lenticularis und den Theil der inneren Kapsel zerstört hatte, welcher sich vorn zwischen Nucleus caudatus und Nucleus lenticularis befindet. Dieser Heerd setzte sich nach unten hin fort in eine dunkelgrau-röthliche gelatinöse Masse, die sclerotisch aussah und sich härtlich anfühlte. Auch hier am Ort der Läsion waren sämmtliche grösseren Gefässe vollständig zart. Der Sehnerv beiderseits zeigte auf seinem Querschnitt eine sehr deutliche Veränderung, nämlich eine ringförmige graue Verfärbung, und dieser entsprachen die mikroskopischen Veränderungen, indem sich Zeichen der Verfettung des Nerven vorfanden. Sonst war im Gross- und Kleinhirn nichts Besonderes zu sehen. An dem Pons aber, und zwar an der Oberfläche in der Höhe des Trigeminus-Austritts, auf das Stratum zonale beschränkt, fand sich ein bohnengrosser Heerd rechterseits, dem sich ein kleinerer linkerseits anschloss. Beide haben, wie Sie bemerken wollen, vollständig das Ansehen sclerotischer Heerde. Die am frischen Präparat vorgenommene mikroskopische Untersuchung ergab indess auch hier nicht eigentlich den Befund der Sclerose, sondern einen solchen, wie man ihn etwa bei secundärer Degeneration findet, wenn dieselbe in graue Degeneration übergeht: kein fibrilläres Gewebe, keine Spinnenzellen, vor Allem keine atrophischen, der Scheide beraubten Nervenfasern, aber sehr reichliche Körnchenzellen und viele wohlerhaltene Nerven: das mikroskopische Bild ist von dem einer beginnenden Erweichung nicht wesentlich verschieden. Es kann aber ein definitives Urtheil über diese Verhältnisse erst abgegeben werden, wenn die Präparate gehärtet und genau untersucht sind. Auch an anderen Stellen des Pons fanden sich kleinere, krankhafter Veränderung verdächtige Stellen.

Auffallend war gegenüber dem leidlichen Blutgehalt beider Substanzen der Hemisphären die überaus grosse Blässe des Hirnstammes. Ich wenigstens habe selten eine so grosse Blässe des Hirnstammes, der Medulla oblongata, des Pons u. s. w. zur Beobachtung bekommen. Auch die Medulla spinalis war ganz blass und von weicher Consistenz. Die Blässe war in den centralen Partien der genannten Theile weniger ausgesprochen, und an verschiedenen Punkten sah man hier selbst kleine Hämorrhagien. Während die Pia cerebralis vollständig klar war, zeigte die Rückenseite der Pia des Rückenmarks ziemlich starke Verdickung im Nacken und Brusttheil. Im Rückenmark selbst, und zwar nur auf die Hinterstränge allein beschränkt, bemerkt man grau verfärbte Stellen fast durch die ganze Länge desselben, welche theils die Fissura mediana posterior unmittelbar begrenzen, theils im mittleren Drittheil der Hinterstränge etwa in der Mitte liegen, also jedenfalls nicht die für die Tabes als bezeichnend gegenwärtig angenommenen Localitäten betreffen.

Ausser diesen Befunden an Schädel und Centralnervensystem, fand sich noch eine Verwachsung des Periostes mit der rechten unebenen Tibia, und eine ausgesprochene syphilitische Veränderung der Leber. Die ganze Leber war in hohem Grade deform geworden durch eine grosse Zahl von Narben, welche nur den serösen Ueberzug und den Theil des Parenchyms betrafen, welcher dicht darunter liegt. Der etwas vergrösserte Herzmuskel war mürb, die Nieren ziemlich stark parenchymatös verfettet. -

Versuchen wir diese Befunde mit den klinisch beobachteten Thatsachen zu erklären, so würden für den Kopfschmerz, den Schwindel, die Hyperostose und sonstigen Veränderungen des Schädels und des Gehirns verantwortlich gemacht werden können. Es könnte wunderbar erscheinen, dass bei dem kleinen derartig hyperostotischen Schädel so hochgradige geistige Intelligenz vorhanden war. Es ist dies aber erklärlich wegen der höchst eigenthümlichen Beschaffenheit des Gehirns und der Unversehrtheit der Rinde und der ernährenden Hirnhäute. Die Hemisphären sind nämlich derartig gestaltet, dass der Theil von der Spitze des Stirnhirns bis zum Sulcus Rolando fast zwei Drittel der Gesammtlänge des Gehirns überhaupt einnimmt, dass also eine überaus beträchtliche Entwicklung des Stirnhirns vorhanden ist. So zeigt sich recht, dass man aus der Beschaffenheit der Schädelkapsel nicht ohne Weiteres auf die geistige Function des Gehirns schliessen kann; es giebt sogar intelligente kleine Dickköpfe wie Sie sehen. Dass der rechtsseitige Hemisphärenheerd keine ausgesprochene, einseitige Lähmung bewirkt hat, kann nicht befremden. Er betraf Theile, die erfahrungsgemäss, wenn sie verletzt werden, keine Lähmungen setzen. Vom Nucleus caudatus Corporis striati weiss man das genau als Resultat 
von Experimenten und pathologischen Erfahrungen. Ich besitze ein Präparat, in welchem durch einen kugelrunden grossen Tuberkel der ganze Kopf des Corp. striat. eingenommen ist, ohne dass bei dem betreffenden Indiv. bei Lebzeiten Lähmung vorhanden gewesen ist. Von dem empfindlicheren Nucleus lenticularis ist nur vorn ein kleiner Theil weggenommen, der mehr die Ganglienmasse als die durchtretenden Nervenfaserzüge betrifft. Es scheint, dass nicht sowohl die Zerstörung einiger Ganglienmasse Lähmungen bewirkt, als vielmehr die Zerstörung der durchtretenden motorischen Fasern. Auch die hier zerstörten vordersten Theile des vordersten Schenkels der inneren Kapsel, deren Function unbekannt ist, scheinen mit der Motilität nichts zu thun zu haben.

Die Sehstörung ist natürlich auf die krankhafte Veränderung der Optici zurückzuführen. $\mathrm{Ob}$ gerade die in der letzten Zeit aufgetretenen Schlingbeschwerden auf die Affection des Pons und angrenzender Theile zu beziehen sind, bin ich ausser Lage bestimmt $\mathrm{zu}$ beantworten.

Für die Psychose wird man in dem pathol.-anatomischen Befund keinen Anhalt suchen wollen. Schon ihre Vergänglichkeit, die vollständige Wiedererlangung des geistigen Gleichgewichts seitens der Patientin, schliesst solchen Gedanken aus. Es giebt keine charakteristische syphilitische Psychose. Nur negativ, insofern hier die Geistestörung keine typische, keine Melancholie, Manie etc. gewesen ist, auch nicht der Entwicklung und dem Verlauf nach, insofern dieselbe mehr den Character allgemeiner Verwirrtheit trug, wie solcher nicht selten allerdings bei materiellen Hirnerkrankungen aber auch bei anämischen Zuständen, Intoxicationen, Blutentmischungskrankheiteu, z. B. Diabetes, Morb. Brightii beobachtet wird, konnte sie, beim Mangel anderweiter Ursachen, als Symptom einer Cerebralaffection bei einer notorisch Syphilitischen einige Beachtung, verdienen.

Noch einige Worte, meine Herren, mit Bezug auf die Störungen im Rückenmark. Es gaben die grauen oder grauröthlichen Stellen in den Hintersträngen mikroskopisch am frischen Präparat auch nur das Bild einer leichten Myelitis. Es reihte sich also hier der Process den im Pons befindlichen an, erschien nur schwächer ausgeprägt. Auch im Rückenmark kann erst nach der Härtung sicher geurtheilt werden über das, was vorliegt; lässt sich heute doch nicht einmal mit Sicherheit entscheiden, ob eine fleckweise Sclerose, ob eine Systemerkrankung vorhanden ist. Handelt es sich indess auch nur um eine Sclerose, so scheint mir ihre Beschränkung auf die Hinterstränge sehr beachtenswerth. Sie wissen von dem Streite, welcher über den Zusammenhang von Tabes und Syphilis entbrannt ist. In unserem Fall ist die luetische Natur sämmtlicher Veränderungen im Centralnervensystem schwer anfechtbar. Wollte man den Befund im Rückenmark (der von der Erkrankung der Häute, im Mitteltheil des mittleren Dritttheils der Hinterstränge $z$. B. sicher nicht abhängt) als einen zufälligen hinstellen, so müsste man wegen der inneren Gleichartigkeit der Processe, dasselbe von den sclerotischen Ponsheerden, von der Grosshimaffection in der Nähe des Chiasma, vom Opticus annehmen.

Es ist also hier Syphilis des Rückenmarkes wie Syphilis des Gehirns und der Optici vorhanden. Andererseits sind gerade die bei der Tabes in Betracht kommenden Antheile der Hinterstränge jedenfalls verschont, wie denn auch bei Lebzeiten der Patientin, was ich ausdrücklich nachholen will, kein Schwanken beim Augenschluss eintrat und die Patellarsehnenreflexe sogar erhöht waren. Indess ist die Sclerose in Bezug auf ihre Localisation unberechenbar. Sie könnte in systemartiger Länge sich einmal anch in den hinteren Wurzelzonen etabliren und dann das Bild einer vielleicht eigenartigen Tabes liefern.

Ich habe endlich noch als bemerkenswerth die, soweit ich bisher untersuchen konnte, intacte Beschaffenheit der Gefässe trotz der Syphilis hervorzuheben. Selbst dort, wo sie den grossen Erweichungsheerd im Gehirn durchzogen, waren sie zwar mit Fett ganz überdeckt, aber nach Entfernung desselben zeigten sie sich im Wesentlichen intact. Die "luetische Erkrankung der Hirngefässe * darf beim Vorhandensein von Erweichungsheerden nicht ausschliesslich als nothwendige Vorbedingung der letzteren gefordert werden. Andere syphilitische Producte z. B. Gummata werden auch nicht durch Gefässerkrankungen, sondern durch das specifische Virus, nach unseren heutigen Anschauungen einen Mikroorganismus, hervorgerufen, welcher, wenn er auch im Blute kreist, doch nicht immer Gefässerkrankungen mit Nothwendigkeit setzen muss, um deletäre Wirkungen zu entfalten.

So mögen denn auch die luetischen Erweichungen und Sclerosen bald durch das Mittelglied der Gefässerkrankung, bald ohne eine solche entstehen. 